\title{
Popularity and Harms of Aural Foreign Bodies: A Descriptive Study of Patients in Baqiyatallah University Hospital, Tehran, Iran
}

\author{
Abolfazl Taheri ${ }^{1}$ \\ Sina Navaei Mehmandari² \\ Mojtaba Shahidi ${ }^{2}$ \\ Hamidreza Mehdizadeh² \\ Seyyed Mohammad Javad Mirlohi ${ }^{3}$ \\ Nasrin Mohammadi Aref $^{3}$ \\ Mahsa Alborzi Avanaki ${ }^{3}$ \\ Mohammad Hossein Khosravi ${ }^{2,4}$
}

\begin{abstract}
Objective: To evaluate the prevalence of external ear complications among Iranian aural foreign body users attending to otolaryngology clinic of our hospital. Methods: In this cross-sectional study patients attending to Otolaryngology clinics of Baqiyatallah hospital were enrolled regardless of their age, gender and reason of attending. Patients between 15 and 60 years of age were included in the present study. Those with positive history of chronic ear diseases, ear surgery, congenital ear disorders, trauma to ear or head and neck region or shock wave trauma were excluded from the study. Demographic information as well as data on chief complaint, educational level, frequency and type of used foreign body and findings of physical examination and Otoscopy by a single otolaryngologist were recorded in a predesigned checklist. Results: Eventually 362 patients ( 232 male and 130 female) with a mean age of $40.32 \pm 16.90$ years underwent analysis. Of all patients $244(67.2 \%)$ were using a kind of aural foreign body frequently and Cotton bud was the most popular (63.5\%) used foreign body among patients. Drying ear canal was the most common (54.9\%) reason of using AFBs among study individuals followed by itching (29.5\%) and pyorrhea (11.06\%). Also 11 (4.5\%) patients were using AFBs as a habit with no specific reason. Itching was the most prevalent symptom reported by both aural foreign body users (78\%) and non-users (45.5\%); however it was significantly higher among AFB users $(p=0.026)$. Also hearing loss was significantly more reported by AFB users $(p=0.033)$. A majority of patients had normal physical examination in both AFB users and non-users group. Inflammation of ear canal was significantly more detected in AFB users $(p=0.004)$. In addition, rate of right ear wax impaction was higher among AFB users $(p=0.016)$. Conclusion: In conclusion we realized that $67.2 \%$ of patients attending to Otolaryngology clinic of our hospital were using a kind of aural foreign bodies and itching was the most common chief complaint of these patients.
\end{abstract}

Keywords: prevalence, foreign bodies, aural foreign bodies, complications

1Department of Otolaryngology, Faculty of Medicine, Baqiyatallah University of Medical Sciences, Tehran, Iran

${ }^{2}$ Student Research Committee, Baqiyatallah University of Medical Sciences, Tehran, Iran

${ }^{3}$ Student Research Committee, Tehran Medical Sciences Branch, Islamic Azad University, Iran

${ }^{4}$ International Otorhinolaryngology Research Association (IORA), Universal Scientific Education and Research Network (USERN), Tehran, Iran

Send correspondence to:

Mohammad Hossein Khosravi

Baqiyatallah Hospital, Mollasadra st., Vanaq sq., Tehran, Islamic Republic of Iran, E-mail: dr.mhkhosravi@gmail.com

Paper submitted to the ITJ-EM (Editorial Manager System) on August 21, 2017;

and accepted on August 30, 2017. 


\section{INTRODUCTION}

Aural Foreign Body (AFB) is defined as every considerable object which is potentially possible to be inserted into the ear canal and is a common presentation to Otolaryngology clinics ${ }^{1}$. Cotton swabs, hairpin and other foreign bodies are frequently used by people for cleaning their ears or as a habit. Ear canal has two parts; external one-third is cartilaginous and internal two-third is bony part which is covered by a thin layer of periosteum and skin and is the most sensitive part of ear to AFBs. The most prevalent clinical finding of patients after removal of foreign body is abrasion of external ear canal skin². This abrasion is a predisposing factor to otitis externa by organisms like Pseudomonas aeruginosa, Staphylococcus aureus, and fungal agents ${ }^{3}$. Also Tympanic membrane could be easily injured and traumatized by a foreign body 4 .

Previous studies have reported that the most common used foreign bodies by children are seeds and cotton tip swabs (CTS) by adults ${ }^{5}$. Foreign body use by people has several known complications which is resulted from the wrong impression about ear wax ${ }^{6,7}$. In addition it has been mentioned that external canal itching is the most prevalent complication of AFBs followed by otitis externa, otomycosis, wax impaction and tympanic membrane rupture ${ }^{8}$.

Conditions that cause harm to external ear are mostly dependent to patients' behavior and are usually preventable; so informing patients about preventing behaviors and possible complications may significantly decrease aural foreign body- related morbidity ${ }^{9-11}$. This action involves proper and accurate perception of aural foreign body use prevalence and its related complications in population.

Despite a variety of conducted studies worldwide for approving the direct relation between prevalence of external ear complications and use of aural foreign bodies, no comprehensive studies have been done in Iran yet. So in the present study we aimed to evaluate the prevalence of external ear complications among Iranian aural foreign body users attending to otolaryngology clinic of our hospital.

\section{MATERIALS AND METHODS}

This cross-sectional study was conducted between May 2016 and June 2016 at Baqiyatallah university hospital, Tehran, Iran. This study was registered at ethics committee of Baqiyatallah University of Medical Sciences.

Patients attending to Otolaryngology clinics of Baqiyatallah hospital regardless of their age, gender and reason of attending were enrolled in the study. Study process was explained for all the patients and a written informed consent was obtained. Patients between 15 and 60 years of age were included in the present study. Those with positive history of chronic ear diseases, ear surgery, congenital ear disorders, trauma to ear or head and neck region or shock wave trauma were excluded from the study.

Demographic information as well as data on chief complaint, educational level, frequency and type of used foreign body and findings of physical examination and Otoscopy by a single otolaryngologist were recorded in a pre-designed checklist.

Data were analyzed using SPSS software version 21 (SPSS Inc., Chicago, IL) for Microsoft Windows. All continuous data are expressed as mean (SD), and categorical variables are expressed as number and percent. Quantitative variables were checked for normality using the Kolmogorov-Smirnoff test. Differences of continuous variables between groups were analyzed using independent $t$-test. A p-value of less than 0.05 was considered as statistically significant.

\section{RESULTS}

Eventually 362 patients ( 232 male and 130 female) with a mean age of $40.32 \pm 16.90$ years underwent analysis. Of all patients $167(46.13 \%)$ had a history of attending to ENT clinic. The most common reason of attending to Otolaryngology clinic was ear problems (40.88\%) followed by nose (27.34\%), pharynx and larynx problems $(12.43 \%)$ as well as headache and sinusitis $(11.43 \%)$ and other causes $(8.01 \%)$. Of study individuals, 116 (32.04\%) had Bachelor of Arts, 35 (9.6\%) had Master of Science or PhD and the remaining had lower educational levels. Of all patients $244(67.2 \%)$ were using a kind of aural foreign body frequently. Figure 1 summarizes the types of aural foreign bodies (AFB) used by patients. Cotton buds were the most popular (63.5\%) used foreign bodies among patients.

Figure 2 shows the frequency of aural foreign body using in study individuals. More than half of the aural foreign body users $(53.2 \%)$ used to use aural foreign

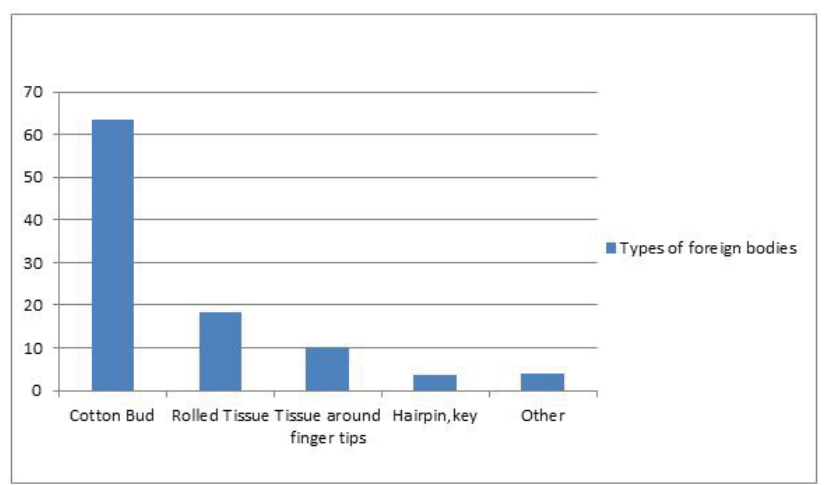

Figure 1. Distribution of used aural foreign bodies among study individuals.

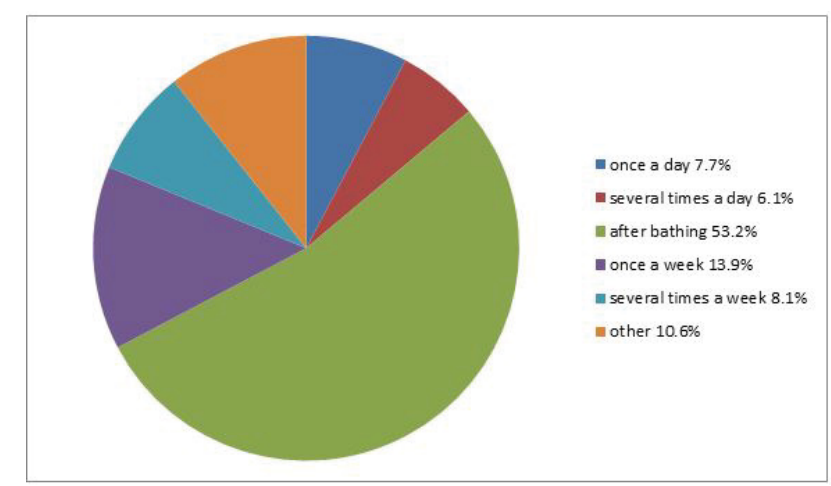

Figure 2. Frequency of aural foreign body using among study individuals. 
bodies after bath. Drying ear canal was the most common (54.9\%) reason of using AFBs among study individuals followed by itching (29.5\%) and pyorrhea (11.06\%). Also $11(4.5 \%)$ patients were using AFBs as a habit with no specific reason.

Among AFB users 126 (51.63\%) had attended to ENT clinic before and $118(48.36 \%)$ had not $(p=0.833)$. A majority (58.56\%) of patients were aware of the fact that using AFBs is harmful upon yet many of them were the ones who used AFBs at the mean time. They mentioned health care workers as the source of information most frequently (51.88\%). Media, Internet and Newspapers were responsible for informing $30.66 \%, 10.37 \%$ and $7.07 \%$ of our patients respectively.

Table 1 shows distribution of symptoms in study individuals. Itching was the most prevalent symptom reported by both aural foreign body users (78\%) and nonusers (45.5\%); however it was significantly higher among AFB users $(p=0.026)$. Also hearing loss was significantly more reported by AFB users ( $p=0.033$ ). Findings of physical examination have been summarized in Table 2. A majority of patients had normal physical examination in both AFB users and non-users group. Inflammation of ear canal was significantly more detected in AFB users ( $p$ $=0.004)$. In addition, rate of right ear wax impaction was higher among AFB users ( $p=0.016)$.

Table 1. Distribution of symptoms reported by study individuals.

\begin{tabular}{cccc}
\hline Signs & FB user (\%) & Non user (\%) & p-value \\
\hline Itching & $98(70.5)$ & $25(45.45)$ & 0.026 \\
Pain & $33(23.745)$ & $11(20)$ & 0.191 \\
Discharge & $56(40.28)$ & $21(38.18)$ & 0.288 \\
Dizziness & $23(16.54)$ & $11(20)$ & 0.114 \\
Hearing Loss & $78(56.11)$ & $23(41.81)$ & 0.033 \\
Feeling Fullness & $27(19.42)$ & $10(18.18)$ & 0.207 \\
Ringing & $19(13.66)$ & $6(10.9)$ & 0.637 \\
Bleeding & $8(5.75)$ & $3(5.45)$ & 0.391 \\
None & $105(43.03)$ & $63(53.38)$ & 0.175 \\
\hline
\end{tabular}

Table 2. Physical examination findings in study individuals.

\begin{tabular}{cccc}
\hline $\begin{array}{c}\text { Physical } \\
\text { examination }\end{array}$ & AFB user (\%) & Non AFB user (\%) & p-value \\
\hline Normal & $130(53.27)$ & $70(59.32)$ & 0.388 \\
Inflammation & $33(28.94)$ & $10(20.83)$ & 0.004 \\
Abrasion & $5(4.38)$ & $1(2.08)$ & 0.327 \\
Otitis & $6(5.26)$ & $1(2.08)$ & 0.022 \\
Right TMP & $3(2.63)$ & $1(2.08)$ & 0.696 \\
Left TMP & $1(0.87)$ & $0(0)$ & 0.747 \\
Otomycosis & $0(0)$ & $0(0)$ & - \\
Otorrhea & $5(4.38)$ & $2(4.16)$ & 0.391 \\
Right wax & $31(27.19)$ & $9(18.75)$ & 0.016 \\
impaction & $28(24.56)$ & $12(25)$ & 0.257 \\
\hline Left wax impaction & & &
\end{tabular}

\section{DISCUSSION}

We found that most (about 67\%) of the patients attending to Otolaryngology clinic of our hospital use aural foreign bodies for a variety of reasons such as itching, humidity, pyorrhea and asymptomatic. The most prevalent reason for visiting was ear problems followed by nasal symptoms. Cotton tip swabs, rolled tissue, tissue around finger tips, hairpin and key were among the most prevalent type of used aural foreign bodies, respectively. In the present study most of the patients were used to use foreign bodies after bath and as a reason of ear canal humidity. More than half of the patients were aware that using aural foreign bodies are harmful and a majority of this awareness was provided by Radio and Television. The most part of the patients were in middle-income category and there was no significant difference between aural foreign body users and non-users for income level. Regarding the symptoms itching and hearing loss were significantly more reported among the foreign body users; however remaining symptoms like dizziness, pain, feeling fullness and ringing were not different between two groups. In physical examination inflammation, external otitis and right ear wax impaction were significantly more present in foreign body users. Other findings such as abrasion, tympanic membrane perforation, otorrhea and left wax impaction were not significantly different between two groups.

Lee et al., in a similar study, reported that about $36 \%$ of study individuals were using a kind of aural foreign bodies which has a lower rate comparing to the present study ${ }^{6}$. In Fasunla et al. and Sheikh et al. study cotton tip swabs were the most prevalent used foreign bodies in patients which are in concordance with the present study $^{12,13}$. Onotai et al. mentioned that cotton buds were being used by $18.6 \%$ of adults as the most common foreign body ${ }^{5}$. Also in Hobson et al. study $53 \%$ of study individuals were used to use cotton buds for cleaning their ear $^{14}$. In agreement with the present study, in Hobson et al. study ear problems were the most common reason for attending to Otolaryngology clinic followed by nasal, pharyngeal and other problems ${ }^{14}$.

In Sheikh et al. study, out of 284 aural foreign body users $25 \%$ of patients had bleeding, $13.8 \%$ had ear canal abrasion and none of the patients had tympanic membrane perforation ${ }^{13}$. In another similar study by Adejbiji et al., 437 patients were foreign body users ${ }^{15}$. Most (63.3\%) of the patients reported hearing loss and pain and tinnitus were reported by $61.3 \%$ and $53.5 \%$ of patients, respectively. The most prevalent $(71.10 \%)$ clinical manifestation of 419 aural foreign body users in Fasunla et al. study was pain in ears ${ }^{12}$.

Kumar et al. have reported itching as the most common (34\%) complication among aural foreign body users followed by external otitis (23\%), otomycosis $(15 \%)$, and external ear canal trauma (9\%). Tympanic membrane perforation was found in $4 \%$ of patients ${ }^{8}$. In accordance with our study, in Adegbiji et al. study most $(75.1 \%)$ of the patients had right ear wax impaction 
which was significantly more prevalent than bilateral wax impaction $^{15}$. In Macknin and colleagues study $7 \%$ of 651 evaluated patients had bilateral wax impaction which had blocked more than $75 \%$ of ear canal ${ }^{16}$.

In a descriptive study by $\mathrm{Al}$-juboori on 224 patients they included patients with positive history of aural foreign body use and those who underwent foreign body extraction at the time. Beads were the most $(30.4 \%)$ prevalent extracted foreign bodies among patients followed by cotton tip swabs (22.3\%) and seeds (13.8\%). In his study $87.5 \%$ of cases had no complications and the common complications were canal abrasion (4.5\%), bleeding (3.6\%), external otitis (3.1\%) and tympanic membrane perforation $(0.9 \%)^{2}$. In a search of 5-year data, Macknin et al. evaluated emergency department visits for aural foreign bodies. Out of 9472 evaluated cases, they reported jewelry as the most common (39.4\%) ear foreign body followed by cotton tip swabs (19\%) and other (28\%) objects $^{16}$.

The present study has some limitations. We did not have complete and comprehensive information on past medical history of patients and also we had a wide range of ages among study individuals.

\section{CONCLUSION}

We realized that $67.2 \%$ of patients attending to Otolaryngology clinic of our hospital were used to use a kind of aural foreign bodies and itching was the most common chief complaint of these patients. Otoscopy was normal in most of the patients and itching was the most prevalent chief complaint. Inflammation and external otitis were significantly more present among aural foreign body users.

\section{Conflict of Interest}

Authors declare, they have no conflict of interest.

\section{REFERENCES}

1. Sterling $M$, Mudd $P$. Destructive otologic foreign body: Dangers of the expanding beard. JAMA Otolaryngol Head Neck Surg. 2016;142(9):919-20.

2. Al-juboori AN. Aural foreign bodies: Descriptive study of 224 patients in Al-Fallujah General Hospital, Iraq. Int J otolaryngol. 2013;2013.

3. Ruddy J, Bickerton RC. Optimum management of the discharging ear. Drugs. 1992;43(2):219-35.

4. Friedman EM. Removal of foreign bodies from the ear and nose. $\mathrm{N}$ Engl J Med. 2016;374(7):e7.

5. Onotai LO, Uriah S. External auditory canal foreign bodies: Clinical profile and management outcomes in Port Harcourt, Nigeria. J Med Res Prac. 2013;2(5):111-5.

6. Lee L, Govindaraju R, Hon S. Cotton bud and ear cleaning-a loose tip cotton bud? Med J Malaysia. 2005;60(1):85.

7. Koçer M, Güldür T, Akarcay M, Miman M, Beker G. Investigation of age, sex and menstrual stage variation in human cerumen lipid composition by high performance thin layer chromatography. J Laryngol Otol. 2008;122(9):881-6.

8. Kumar S, Ahmed S. Use of cotton buds and its complications Journal of Surgery Pakistan (International). 2008;13(3):137-8.

9. Browning G. Ear wax. BMJ Clin Evid. 2006;10:504.

10.Clegg A, Loveman E, Gospodarevskaya E, Harris P, Bird A, Bryant $J$, et al. The safety and effectiveness of different methods of ear wax removal: A systematic review and economic evaluation. Health Technol Assess. 2010;14(28):1-191.

11. Oron Y, Zwecker-Lazar I, Levy D, Kreitler S, Roth Y. Cerumen removal: Comparison of cerumenolytic agents and effect on cognition among the elderly. Arch Gerontol Geriatr. 2011;52(2):228-32.

12.Fasunla $\mathrm{J}$, Ibekwe T, Adeosun A. Preventable risks in the management of aural foreign bodies in Western Nigeria. Internet $J$ Otorhinolaryngol. 2006;7:1-4.

13. Sheikh MS, Sheikh MA, Shafique MA. Frequency of three complications (bleeding, laceration of external auditory canal and perforation of tympanic membrane) in patients with aural foreign bodies. Med Forum. 2015;26(1):29-32.

14. Hobson JC, Lavy JA. Use and abuse of cotton buds. J R Soc Med. 2005;98(8):360-1.

15.Adegbiji WA, Alabi BS, Olajuyin OA, Nwawolo C. Earwax impaction: Symptoms, predisposing factors and perception among Nigerians. $J$ Family Med Prim Care. 2014;3(4):379-82.

16. Macknin ML, Talo H, Medendorp SV. Effect of cotton-tipped swab use on earwax occlusion. Clin Pediatr. 1994;33(1):14-8. 